\title{
Proof Automation in the Theory of Finite Sets and Finite Set Relation Algebra
}

\author{
Maximiliano Cristiá ${ }^{1}$, Ricardo D. Katz ${ }^{2}$ and Gianfranco \\ $\operatorname{RossI}^{3}$ \\ ${ }^{1}$ Universidad Nacional de Rosario and CIFASIS, Argentina \\ ${ }^{2}$ CIFASIS-CONICET, Argentina \\ ${ }^{3}$ Università di Parma, Italy \\ Email: cristia@cifasis-conicet.gov.ar,katz@cifasis-conicet.gov.ar,gianfranco.rossi@unipr.it
}

\begin{abstract}
$\{\log \}$ ('setlog') is a satisfiability solver for formulas of the theory of finite sets and finite set relation algebra (FS\&RA). As such, it can be used as an automated theorem prover (ATP) for this theory. $\{l o g\}$ is able to automatically prove a number of FS\&RA theorems, but not all of them. Nevertheless, we have observed that many theorems that $\{\log \}$ cannot automatically prove can be divided into a few subgoals automatically dischargeable by $\{\log \}$. The purpose of this work is to present a prototype interactive theorem prover (ITP), called $\{\log \}$-ITP, providing evidence that a proper integration of $\{l o g\}$ into world-class ITP's can deliver a great deal of proof automation concerning FS\&RA. An empirical evaluation based on 210 theorems from the TPTP and Coq's SSReflect libraries shows a noticeable reduction in the size and complexity of the proofs with respect to Coq.
\end{abstract}

Keywords: $\{\log \}$; Set theory; Automated proofs; Constraint logic programming

\section{INTRODUCTION}

Interactive theorem proving (ITP) [1] is increasingly used in the formalization and proof of results of mathematics and logic, and also a widely used approach to formal verification of hardware and software. ITP's such as Coq [2, Isabelle/HOL [3] and HOL Light [4] vary in the level of expressivity and automation, but typically support rich specification languages including higher-order logic or dependent type theory. Since interactive theorem proving is labor intensive, thus costly and limited, much research has been devoted to the development of automated reasoning.

SMT solvers 5 implement decision procedures for the satisfiability problem of formulas of specific theories. Since they have significantly improved their power in the last decades, it has become increasingly common for ITP's the use of SMT solvers as efficient automated theorem provers (ATP) for the corresponding theories. As a consequence, users of mainstream ITP's can call ATP's 6, 7, and SMT solvers 8, 9, to automatically advance their proofs. These ad-ons exploit the idea of mixing interactive and automated proof steps. Our proposal fits in this line of work as we propose to integrate $\{l o g\}$ as a special purpose ATP into ITP's.

$\{\log \}$ is a satisfiability solver accepting an input language at least as expressive as the class of full set relation algebras on finite sets (FS\&RA) [10]. FS\&RA essentially corresponds to the first-order fragment of formal notations such as Alloy [11, B [12] and Z [13] restricted to finite sets. In consequence, this input language can be used as a specification language for a large class of software systems and $\{\log \}$ as a tool to reason about them.

$\{\log \}$ can automatically prove $97 \%$ of the theorems on Boolean algebra (BOO), relation algebra (REL) and set theory (SET) gathered in the TPTP library 14 that can be expressed in its input language (in $.3 \mathrm{~s}$ each in average), see 15. Since the equational theory of FS\&RA is undecidable [16], $\{\log \}$ cannot decide the satisfiability of all the formulas it accepts. Moreover, $\{\log \}$ can take too long to decide the satisfiability of some formulas. For example, it takes a long time to prove the following result:

$$
\begin{aligned}
f & \in A \rightarrow B \wedge \operatorname{ran} f=B \wedge g \in B \rightarrow C \\
& \wedge h \in B \rightarrow C \wedge f \circ g=f \circ h \Longrightarrow g=h
\end{aligned}
$$

where $A, B$ and $C$ denote any finite sets, $A \rightarrow B$ denotes the set of all (finite) functions from $A$ to $B$ (in this context a function is a binary relation where no two ordered pairs have the same first component) and ran $f$ is the range of $f$. Nevertheless, since $g=h \Longleftrightarrow$

\footnotetext{
${ }^{4} \mathrm{By}$ an abuse of language, from now on we will say that $\{\log \}$ can or cannot prove a theorem to mean that it can decide or not the satisfiability of its negation (see Remark 2 below).
} 
$g \subseteq h \wedge h \subseteq g$, the proof of (T1) can be reduced to the proofs of the following two implications, on which $\{\log \}$ spends a few seconds:

$$
\begin{aligned}
& f \in \star \rightarrow \star \wedge \operatorname{ran} f=B \wedge g \in B \rightarrow \star \\
& \wedge h \in \star \rightarrow \star \wedge f \circ g=f \circ h \Longrightarrow g \subseteq h \\
& f \in \star \rightarrow \star \wedge \operatorname{ran} f=B \wedge g \in \star \rightarrow \star \\
& \quad \wedge h \in B \rightarrow \star \wedge f \circ g=f \circ h \Longrightarrow h \subseteq g
\end{aligned}
$$

Here $\star$ means that the corresponding hypotheses can be dropped (for example, $f \in \star \rightarrow \star$ says that it does not matter what the domain and range of $f$ are). Thus, by dividing the proof of (T1) into subgoals and by dropping the appropriate hypotheses in each, $\{\log \}$ can automatically do the rest.

We have noticed that in practice the approach above succeeds on proving many results that $\{\log \}$ cannot automatically prove. As a consequence, in this paper we present $\{l o g\}$-ITP, a prototype interactive theorem prover where users can enter any $\{\log \}$ formula and interactively prove it. More precisely, in $\{\log \}$-ITP users can divide the proof into subgoals, drop hypotheses and call $\{\log \}$ to perform the actual mathematical steps. This follows the way other tools, such as Atelier B [17] and the Coq's why3 [18] tactic, work. We point out that $\{\log \}$-ITP is just a vehicle to provide evidence that a proper integration of $\{\log \}$ 's rewriting system into world-class ITP's can deliver a great deal of proof automation concerning FS\&RA.

In order to validate our proposal in practice we perform a number of proofs with $\{\log \}$-ITP and Coq. On these theorems $\{\log \}$ either does not terminate or takes a very long time to do it. This comparison shows promising results as Coq proofs are harder and longer than $\{\log \}$-ITP's (see Section 4 for details).

As SMT solvers, $\{\log \}$ generates a solution when it determines that a formula is satisfiable. Indeed, $\{\log \}$ provides a finite representation of all the (possibly infinitely many) solutions [10. In the context of integrating $\{\log \}$ into an ITP, this means that if $\{\log \}$ is called to advance a proof but it happens that the goal is not provable from the premises, a counterexample is generated. This counterexample might help the user to adjust the theorem or the theory which contains it. QuickChick has been proposed as a tool to decrease the number of failed proof attempts in Coq by generating counterexamples before a proof is attempted [19]. This tool relies on a random counterexample generator. Although $\{\log \}$ counterexamples are generated in a deductive fashion, it is also more limited as it works only for a specific theory.

Let us finally mention that traditional ATP's such as E prover [20] and Vampire 21] can automate proofs of FS\&RA. Since they can efficiently solve many FOL problems, they work by encoding set theory in (most often) untyped first-order logic. One of the simplest encodings applies extensionality and rewrites away all definitions, thus arriving at formulas based on set membership. However, these encodings must deal with typing information when sets do not have the same set support. The easiest way to deal with this issue is to omit all type information, but this approach is unsound. Another way to deal with types is to annotate terms with type tags or guards. This considerably increases the size of the problems passed to generic ATP, with a dramatic impact on their performance 22, 23. Another approach to proof automation in set theory is to use polymorphic provers. Our work follows this approach as $\{l o g\}$ can be seen as a specialized prover for polymorphic set theory. The empirical assessment presented in this paper confirms the results reported by other polymorphic provers $23,24,25,26,27$.

This paper is structured as follows. The logic language supported by $\{\log \}$ and some of its main features are presented in Section 2, Section 3 describes $\{l o g\}$-ITP which is empirically evaluated in Section 4 We give our final conclusions in Section 5 .

\section{2. $\{L O G\}:$ A SATISFIABILITY SOLVER FOR FINITE SETS AND RELATIONS}

In this section we provide a brief, informal introduction to the $\{\log \}$ system 29]. A formal presentation of $\{\log \}$ 's language can be found in Appendix A deeper presentations can be found elsewhere [10, 15, 28].

\subsection{The $\{\log \}$ language}

$\{\log \}[29$ is a satisfiability solver implemented in Prolog whose input language is denoted $\mathcal{L}_{\mathcal{B R}}$. This is a multisorted first-order predicate language with two distinct sorts: the sort Set of all the terms which denote sets (including binary relations) and the sort $\mathrm{O}$ of all the other terms. Thus, we do not introduce distinct sorts for sets and binary relations. Binary relations are just sets of ordered pairs. This allows sets and relations to be freely mixed; in particular all set operators are directly applicable to binary relations.

Note 1 (Background on binary relations). Let $R$ and $S$ be binary relations, and $A$ a set. Then, we define following relational operators: relational composition, $R \circ S=\{(x, z) \mid \exists y((x, y) \in R \wedge(y, z) \in S)\}$; converse (or inverse) of $R, R^{\smile}=\{(y, x) \mid(x, y) \in R\}$; the identity relation on $A$, id $A=\{(x, x) \mid x \in A\}$; domain of $R, \operatorname{dom} R=\{x \mid \exists y((x, y) \in R)\}$; range of $R, \operatorname{ran} R=\operatorname{dom} R^{\smile}$; domain restriction of $R$ on $A, A \triangleleft R=\operatorname{id}(A) \circ R$; range restriction of $R$ on $A$, $R \triangleright A=R \circ \operatorname{id}(A)$; domain anti-restriction of $R$ on $A$, $A \triangleleft R=R \backslash(A \triangleleft R)$; range anti-restriction of $R$ on $A$, $R \triangleright A=R \backslash(R \triangleright A)$; relational image of $A$ through $R, R[A]=\operatorname{ran}(A \triangleleft R)$; and relational overriding of $R$ by $S, R \oplus S=(\operatorname{dom} S \triangleleft R) \cup S$. A binary relation is a (partial) function if no two of its ordered pairs have the same first component. Given that functions are just binary relations, all relational operators can be applied to functions. 
In $\mathcal{L}_{\mathcal{B R}}$ set operators are encoded as constraints over the domain of finite hybrid sets. For example: un $(A, B, C)$ is a constraint interpreted as $C=A \cup B$. $\{\log \}$ implements a wide range of set and relational operators (cf. Note 2). For instance, $\in$ is a constraint interpreted as set membership; = is set equality; $\operatorname{dom}(R, D)$ corresponds to $\operatorname{dom} R=D ; A \subseteq B$ corresponds to the subset relation; $\operatorname{comp}(R, S, T)$ is interpreted as $T=R \circ S$; and $\operatorname{apply}(F, X, Y)$ is equivalent to $\operatorname{pfun}(F) \wedge[X, Y] \in F$, where $\operatorname{pfun}(F)$ constrains $F$ to be a (partial) function. Formulas in $\{\log \}$ are conjunctions $(\wedge)$ and disjunctions $(\vee)$ of constraints. Negation is introduced by means of socalled negated constraints. For example nun $(A, B, C)$ is interpreted as $C \neq A \cup B$ and $\notin$ as the negation of $\notin$. In general, if $\pi$ is a constraint, $n \pi$ corresponds to its negated form. For formulas to fit inside the decision procedures implemented in $\{\log \}$, users must only use this form of negation.

In turn, terms can be either uninterpreted Herbrand terms (as in Prolog) or set terms, i.e., terms with the following form and interpretation: $\emptyset$ to denote the empty set; $\{x \sqcup A\}$, called extensional set, which is interpreted as $\{x\} \cup A$, where $A$ is a set term; and $A \times B$ to represent the Cartesian product between the sets denoted by set terms $A$ and $B$.

In $\{\log \}$ sets are always finite and untyped and they are allowed as set elements (i.e., sets can be nested). As the second argument of an extensional set can be a variable, sets in $\{l o g\}$ can be unbounded.

Note 2 (Binary relations and expressivenes). $\mathcal{L}_{\mathcal{B R}}$ turns out to be at least as expressive as the class of full set relation algebras on finite sets. A full set relation algebra [16] over a base set $A$, denoted $\mathfrak{R}(A)$, is the relation algebra where relations are subsets of $A \times A$. A mapping from formulas of a $\mathfrak{R}(A)$ with $A$ finite to $\mathcal{L}_{\mathcal{B R}}$ formulas can be easily defined [10]. The class of full set relation algebras on finite sets is the class of relation algebras $\mathfrak{R}(A)$ where $A$ is a finite set. Further, $\mathcal{L}_{\mathcal{B R}}$ allows for the definition of the class of full set heterogeneous relation algebras on finite sets. An heterogeneous relation algebra deals with relations between two arbitrary sets $A$ and $B$, i.e. with subsets of $A \times B$ [33. We use the acronym FS\&RA to denote such a class, for any $A$ and $B$ finite.

The class of full set heterogeneous relation algebras roughly corresponds to the first-order fragment of formal notations such as Alloy [11, B [12] and Z [13. A large class of programs can be specified within this fragment. The limitation of $\mathcal{L}_{\mathcal{B R}}$ to finite sets is not so severe as many programs operate only on finite data structures. Therefore, $\mathcal{L}_{\mathcal{B R}}$ can be used as a specification language for a large class of software systems and $\{\log \}$ as a tool to reason about them [31, 34, 35, 30, 32].

\subsection{A rewriting system for $\mathcal{L}_{\mathcal{B R}}$}

$\{l o g\}$ implements a rewriting system for $\mathcal{L}_{\mathcal{B R}}$ formulas, called $S A T_{\mathcal{B R}}$, whose global organization is shown in Algorithm 1. Basically, $S A T_{\mathcal{B R}}$ uses two procedures: sort_infer and STEP.

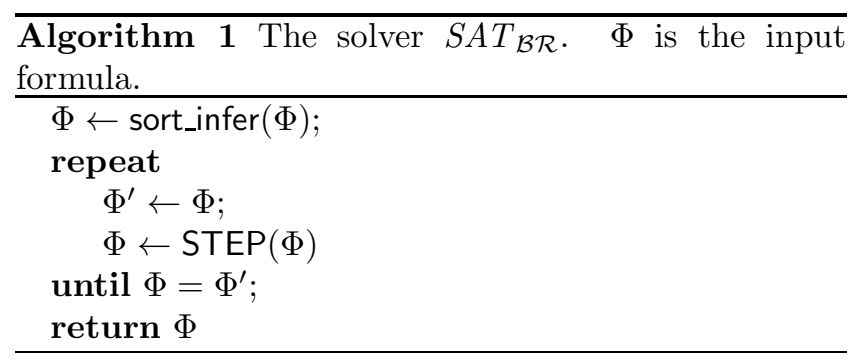

$\mathcal{L}_{\mathcal{B R}}$ does not provide variable declarations. For this reason, sort_infer $(\Phi)$ automatically adds either a set or a rel constraint for each variable $X$ in $\Phi$ which is required to represent, respectively, either a set or a binary relation according to the intended interpretation of the terms or constraints where $X$ occurs.

STEP applies specialized rewriting procedures to the current formula $\Phi$ and returns either false or a modified formula. Each rewriting procedure applies a few non-deterministic rewrite rules which reduce the syntactic complexity of $\mathcal{L}_{\mathcal{B R}}$ constraints of one kind. The execution of STEP is iterated until a fixpoint is reached, i.e., the formula is irreducible. STEP returns false whenever (at least) one of the procedures in it rewrites $\Phi$ to false.

The rewriting procedures implemented in $\{\log \}$ can be divided into two classes: those for set constraints and those for relational constraints. The former were introduced in 36] and extensively discussed from then on. They constitute the base for a decision procedure for finite sets based on set unification and set constraint solving. The latter were introduced more recently [10, 15. Roughly, there are 50 rewriting procedures adding up 175 rewrite rules.

Here we just show some of the most representative rewrite rules in Figure 10 (the reader can find a comprehensive list online [37]). Note that these rules are recursive. Rule (11) finds all possible solutions for the equality between two non-empty extensional sets. The second and third disjuncts take care of duplicates in the right- and left-hand side terms, respectively, while the last disjunct takes care of permutativity of the set constructor $\{\cdot \sqcup \cdot\}$. Specifically, the last disjunct can be read as ' $y$ must belong to $A, x$ must belong to $B$ and there exists a set $N$ containing the remaining elements of both $A$ and $B^{\prime}$. In turn, rule (2) finds all possible solutions of a set union operation when the result is a non-empty extensional set, where $N, N_{1}$ and $N_{2}$ are new variables (implicitly existentially quantified). Note that set unification is used to avoid possible repetitions of $t$ in $C$. Also observe that the disjunction captures the three possible solutions: $t$ belongs to $A, t$ belongs 


$$
\begin{gathered}
\{x \sqcup A\}=\{y \sqcup B\} \rightarrow \\
x=y \wedge A=B \\
\quad \vee x=y \wedge\{x \sqcup A\}=B \\
\quad \vee x=y \wedge A=\{y \sqcup B\} \\
\quad \vee A=\{y \sqcup N\} \wedge\{x \sqcup N\}=B \\
u n(A, B,\{t \sqcup C\}) \rightarrow \\
\{t \sqcup C\}=\{t \sqcup N\} \wedge t \notin N \\
\wedge\left(A=\left\{t \sqcup N_{1}\right\} \wedge u n\left(N_{1}, B, N\right)\right. \\
\quad \vee B=\left\{t \sqcup N_{1}\right\} \wedge u n\left(A, N_{1}, N\right) \\
\quad \vee A=\left\{t \sqcup N_{1}\right\} \\
\left.\quad \wedge B=\left\{t \sqcup N_{2}\right\} \wedge \text { un }\left(N_{1}, N_{2}, N\right)\right) \\
\operatorname{inv}(R,\{(y, x) \sqcup S\}) \rightarrow \\
R=\{(x, y) \sqcup N\} \wedge \operatorname{inv}(N, S)
\end{gathered}
$$

FIGURE 1. Representative rewriting rules

to $B$ and $t$ belongs to $A$ and $B$. Finally, rule (3) finds the binary relation whose converse is a non-empty extensional relation in a very simple way.

The rewriting system implemented by $\{\log \}$ has been proved to be a semi-decision procedure [10. More precisely, it has been proved that: a) when Algorithm 1 terminates, the returned formula preserves the set of solutions of the input formula; b) the returned formula is false if and only if the input formula is unsatisfiable; and c) if the returned formula is not false it is trivial to calculate one of its solutions (basically by substituting all set variables by the empty set). In this context, a 'solution' is an assignment of values to all the free variables of the formula. Furthermore, when $\{\log \}$ terminates, it has the ability to produce a finite representation of all the (possibly infinitely many) solutions of the input formula, in the form of a finite disjunction of $\mathcal{L}_{\mathcal{B R}}$ formulas. In other words, whenever $\{\log \}$ terminates, it either produces a proof of unsatisfiability or a finite representation of all the solutions.

\subsection{Using $\{\log \}$}

Users interact with $\{\log \}$ by simply entering a formula; there are no user commands. If the formula is unsatisfiable $\{\log \}$ will simply return false and if it is satisfiable it will return a finite representation of all its solutions.

Example 1. For example, the following is a satisfiable formula (note that binary relations can be freely combined with extensional sets, and set operators can take relations as arguments):

$$
\begin{aligned}
& u n(A, B,\{(1,1),(h, 3) \sqcup C \times D\}) \\
& \quad \wedge i d(E, A) \wedge i n v(B, B) \wedge 1 \notin E
\end{aligned}
$$

The relevant part of a solution returned by $\{\log \}$ is:

$$
\begin{aligned}
A=\left\{(3,3) \sqcup N_{3}\right\}, B=\left\{(1,1) \sqcup N_{2}\right\}, & \\
h=3, E= & \left\{3 \sqcup N_{1}\right\} \\
\text { Constraint: } & 3 \notin C, \text { un }\left(N_{2}, N_{3}, C \times D\right), i d\left(N_{1}, N_{3}\right), \\
& i n v\left(N_{2}, N_{2}\right), \ldots
\end{aligned}
$$

where $N_{i}$ are fresh variables. That is, each solution is composed of a (possibly empty) conjunction of equalities between variables and terms and a (possibly empty) conjunction of constraints. The conjunction of constraints is guaranteed to be trivially satisfiable.

In this context, $\{\log \}$ can be used as a set-based, constraint-based programming language. Users can give values to what they consider to be input variables in the formula and $\{\log \}$ will return values for the remaining variables. For instance, if (4) is thought as a program where $A$ and $B$ are inputs and the user enters (44) conjoined with $A=\{(2,2),(3,3)\} \wedge B=$ $\{(1,1),(1,2),(2,1)\}$, the answer will be $h=3, C=D=$ $\{1,2\}, E=\{2,3\}$. In $\{\log \}$, formulas are programs.

Given that $\{\log \}$ is a satisfiability solver we can use it also as an automated theorem prover. To prove that formula $\Phi$ is a theorem, $\{\log \}$ has to be called on $\neg \Phi$ waiting an false answer, meaning that $\neg \Phi$ is unsatisfiable (and thus $\Phi$ is a theorem).

EXAMPLE 2. We can prove that set intersection is commutative by asking $\{\log \}$ to prove the following formula is unsatisfiable:

$$
\operatorname{inters}(A, B, C) \wedge \operatorname{inters}(B, A, D) \wedge C \neq D
$$

As there are no finite sets satisfying this formula, $\{\log \}$ returns false. The formula can also be written as:

$$
\text { inters }(A, B, C) \wedge \operatorname{ninters}(B, A, C)
$$

All these properties along with programming facilities not discussed in this paper [29], make $\{\log \}$ a versatile verification tool [31, 34, 35, 30, 32.

\section{AUTOMATING COMPLEX FS\&RA PROOFS}

As we have pointed out, $\{\log \}$ may not terminate or may take a very long time when it is used to prove some theorems of FS\&RA. However, we have observed that in practice the proofs of many of such theorems can be divided into a few subgoals each of which can be automatically and quickly discharged by $\{\log \}$. In fact, these proofs follow a recurring pattern: divide the proof by introducing some assumptions, drop zero or more hypotheses in each subgoal and call $\{\log \}$ to do the hard, annoying mathematical work. This would imply that complex theorems of FS\&RA can be easily proved by calling $\{\log \}$ at the right points.

In order to provide evidence of these observations, we have developed a proof-of-concept ITP on top of $\{\log \}$ 
that we call $\{\log \}$-ITP. This is a freely available +500 LOC Prolog program 38, that allows users to declare a $\{\log \}$ formula as a theorem and attempt to prove it interactively through some proof commands. First, we will show a typical proof using $\{l o g\}$-ITP, and then we will give technical details about the proof system.

REMARK 1 (Limitations). It is important to bear in mind that $\{\log \}$-ITP is intended to be an ITP only for theorems of FS\&RA and only to empirically validate our proposal. This means that it cannot be compared in no way with general-purpose ITP's such as Coq or Isabelle/HOL.

\subsection{A typical proof}

(T1) is declared as a $\{l o g\}$-ITP theorem with the theorem command:

theorem $(T 1$,

$$
\begin{aligned}
& \operatorname{pfun}(f) \wedge f \subseteq A \times B \wedge \operatorname{dom}(f, A) \wedge \operatorname{ran}(f, B) \\
& \wedge \operatorname{pfun}(g) \wedge g \subseteq B \times C \wedge \operatorname{dom}(g, B) \\
& \wedge \operatorname{pfun}(h) \wedge h \subseteq B \times C \\
& \wedge \operatorname{dom}(h, B) \wedge \operatorname{comp}(f, g, N) \wedge \operatorname{comp}(f, h, N), \\
& g=h)
\end{aligned}
$$

where the first parameter is just a name for the theorem, the second one is a (possibly empty) conjunction of hypotheses and the third one is the thesis, both entered as $\{\log \}$ formulas. Note that $f \in A \rightarrow B$ is encoded in $\{\log \}$ as $p f u n(f) \wedge f \subseteq A \times B \wedge \operatorname{dom}(f, A)$ and that $f \circ g=f \circ h$ is encoded as two comp constraints yielding the same result $(N)$.

As we have said, an automated proof of (T1) would take a long time. However, the interactive proof shown in Figure 2 takes only a few seconds of computing time. There, $\Gamma$ represents the hypotheses of (T1). The proof starts with the rewrite command which splits the proof into the two subgoals shown in Figure 2. Attempting to use $\{\log \}$ to prove these subgoals by means of the command prove would consume as much time as the proof of the initial goal because they are essentially the same. As the proof of these two subgoals is symmetric, we will explain in detail only the first one. In this case the user can use the following drop command, which expects a list of $\{\log \}$ constraints:

$$
\begin{aligned}
\operatorname{drop}([f & \subseteq A \times B, \operatorname{dom}(f, A), \\
g & \subseteq B \times C, h \subseteq B \times C, \operatorname{dom}(h, B)])
\end{aligned}
$$

These constraints are expected to be part of $\Gamma$ in which case they are removed from it, thus yielding the following hypotheses:

$$
\begin{aligned}
& \operatorname{pfun}(f) \wedge \operatorname{ran}(f, B) \wedge \operatorname{pfun}(g) \wedge \operatorname{dom}(g, B) \\
& \wedge \operatorname{pfun}(h) \wedge \operatorname{comp}(f, g, N) \wedge \operatorname{comp}(f, h, N)
\end{aligned}
$$

called $\Gamma_{1}$ in Figure 2, Now, prove discharges the current subgoal in a few seconds. Since in this case $\{\log \}$ succeeds in proving the goal, the system shows to the user the remaining subgoal. A similar course of action is taken to discharge the remaining subgoal, where a different list of constraints is passed in to the drop command.

In Section 3.3 we discuss some aspects of this proof and present the complete proof script in (6).

\subsection{Proof commands}

In this section we present in detail the main proof commands of $\{\log \}$-ITP (see Figure 3). Some of them are direct implementations of well-known inference rules while others implement a few such rules in a single proof step.

REMARK 2 (Interfacing with $\{\log \}$ ). As we already said, as $\{\log \}$ is a satisfiability solver, it can be used as an ATP. Indeed, if $\{\log \}$ finds that formula $\varphi$ is unsatisfiable in FS\&RA, then $\neg \varphi$ is a theorem (in FS\&RA). Thus, when $\{\log \}$ is used as a back-end system for an ITP, formulas must be negated before sending them from the ITP to $\{\log \}$. However, with the intention to simplify the presentation, we are not going to mention this negation process in the remaining of the paper. This implies that, for example, when in the command called prove (Figure 3) we say that $\Gamma \Longrightarrow \Delta$ is sent to $\{\log \}$ it actually means that its negation $\Gamma \wedge \neg \Delta$ is sent to it.

Concerning Figure 3, the assume command can be seen as the implementation of a special case of the Cut rule; cases corresponds to conjunction introduction; and drop is a specialized version of the Weakening rule where the antecedent is weakened in order to deliver to $\{\log \}$ exactly the necessary hypotheses that yield the consequent.

define waits for a constraint $\pi \in\{u n, i n v, i d, c o m p\}$. The last argument of $\pi$ is expected to be a new variable and all the others must be variables in the current scope (of the proper sort). For instance, if in the current scope $R$ is a binary relation then the user can issue define $(\operatorname{inv}(R, S))$, where $S$ is a new variable, in which case $\operatorname{inv}(R, S)$ is added as a new hypothesis. This is sound because what we are doing is no more than asserting that the converse of $R$ is called $S$. Now the user can make assumptions on $S$. For example, assume $\operatorname{pfun}(S))$, which means that $S$ (i.e., the converse of $R$ ) is a function. Without such a command it would be impossible to consider assumptions on expressions assembled from variables in the current scope, as in $\{l o g\}$-ITP set and relational operations are represented as predicates.

The prove command simply calls $\{\log \}$ on the current subgoal. In this case, there are three possible behaviors: a) $\{\log \}$ answers that the current subgoal is indeed valid and so it is proved and the next one (if any) is shown to the user; $b)\{\log \}$ answers that there is a counterexample (for instance if too many hypotheses 


$$
\operatorname{drow} \frac{\operatorname{prove} \frac{\{\log \}}{\Gamma_{1} \vdash g \subseteq h}}{\Gamma \vdash g \subseteq h} \quad \text { drop } \frac{\text { prove } \frac{\{\log \}}{\Gamma_{2} \vdash g \subseteq h}}{\Gamma \vdash h \subseteq g}
$$

FIGURE 2. $\{\log \}$-ITP proof of theorem T1 or (T1)

$$
\begin{aligned}
& \operatorname{assume}(\varphi) \frac{\Gamma, \varphi \vdash \Delta \quad \Gamma \vdash \varphi}{\Gamma \vdash \Delta} \quad \operatorname{cases} \frac{\Gamma \vdash \varphi, \Delta \quad \Gamma \vdash \xi, \Delta}{\Gamma \vdash \varphi \wedge \xi, \Delta} \quad \operatorname{drop}(\varphi) \frac{\Gamma \vdash \Delta}{\Gamma, \varphi \vdash \Delta} \\
& \operatorname{define}(\pi) \frac{\Gamma, \pi(\ldots, n) \vdash \Delta}{\Gamma \vdash \Delta} \quad \quad \text { prove } \frac{\operatorname{set} \log (\Gamma \Longrightarrow \Delta)}{\Gamma \vdash \Delta} \\
& \underline{\Gamma,(v=t)[n / x] \vdash \varphi[n / x]} \quad \quad \frac{\Gamma,(w=u)[n / x] \vdash \xi[n / x]}{\Gamma \vdash(w=u} \\
& \Gamma \vdash(v=t \Longrightarrow \varphi)[n / x] \quad \quad \frac{\Gamma}{\Gamma \vdash(w=u \Longrightarrow \xi)[n / x]} \\
& \Gamma \vdash \forall x: v=t \Longrightarrow \varphi \quad \Gamma \vdash \forall x: w=u \Longrightarrow \xi \\
& \text { rewrite } \frac{\Gamma \vdash(\forall x: v=t \Longrightarrow \varphi) \wedge(\forall x: w=u \Longrightarrow \xi)}{\Gamma \vdash \pi(v, w)}
\end{aligned}
$$

FIGURE 3. Main $\{\log \}$-ITP proof commands as inference rules

have been dropped) in which case a proper error message is printed; and c) $\{\log \}$ takes too long and the user decides to interrupt the command. In $b$ and $c$ the proof is unchanged. In $b$ users can execute command counterex to get a counterexample witnessing why the goal failed (recall the discussion on the generation of counterexamples in the introduction).

rewrite calls $\{\log \}$ to rewrite the thesis; that is $\{\log \}$ is called to apply a rewrite rule to the thesis (eg. one of the rules of Figure 11) thus generating one or more new goals to prove - this last case occurs when the rewrite rule is nondeterministic. The thesis is assumed to be a single constraint. Each of these new goals should be simpler to prove than the orginal one. For each new goal generated by the rewrite rule, rewrite performs three proof steps in one (see Figure 3):

1. It applies conjunction introduction to the goal. If the subgoal is a conjunction of constraints, then the user will prove one after the other.

2. It applies universal introduction on each subgoal generated in step 1. As the new goal will in general be a universally quantified formula, these quantified variables are 'introduced'.

3. It applies conditional introduction on each subgoal generated in step 1. As in the previous step, the new goal will in general be a conditional and so hypotheses are 'introduced' as well.

For example, if $\Gamma \vdash p f u n(f)$ is the current goal, the net effect of rewrite is shown in Figure 4 where $x, y, z, v$ and $N$ are new variables. If, for instance, $\{\log \}$ does not terminate on $\Gamma \vdash p f u n(f)$, after rewrite the user has two simpler goals to prove. In particular, the one on the right most often can be automatically and quickly discharged with prove. In Figure 4 rewrite produces those two subgoals because in $\mathcal{L}_{\mathcal{B R}}$ we have:

$$
\begin{aligned}
\operatorname{pfun}(f) & \Leftrightarrow \\
& (\forall v: v \in f \Rightarrow \operatorname{pair}(v)) \\
& \wedge(\forall x, y, z:(x, y) \in f \wedge(x, z) \in f \Rightarrow y=z)
\end{aligned}
$$

where $v \in f$ is equivalent to $f=\{v \sqcup N\}$ for some new variable $N$, which yields the equalities seen in Figure 4 Then, when conjunction, universal quantification and implication are introduced as in Figure 3, we get the result shown in Figure 4

Actually, the inference rule given for rewrite in Figure 3 is a simplification of the real rule. Here we assume that the current thesis is a constraint $\pi$ depending on two variables ( $v$ and $w$ ), which when rewritten by $\{\log \}$ delivers a conjunction of two universal formulas (in the real case it can be any number of them). These formulas have all the same form equalities $\Longrightarrow$ predicate, where equalities is a (possibly empty) conjunction of equalities of the form $\mathrm{var}=$ term where var is one of the variables on which $\pi$ depends on; and predicate is a (possibly empty) conjunction of $\mathcal{L}_{\mathcal{B} \mathcal{R}}$ constraints. In Figure 3 we assume that the equalities in each conjunct have exactly one equality.

It is important to remark that $\{\log \}$-ITP does not need a command, for instance, to perform equality substitution because this is performed by $\{\log \}$ when prove is executed. In effect, if prove is issued, for example, on $\Gamma, f=\{v \sqcup N\} \vdash \operatorname{pair}(v)$, then $\{\log \}$ will substitute $f$ by $\{v \sqcup N\}$ in $\Gamma$.

\subsection{Discussion}

As can be seen in Figure 3, many $\{l o g\}$-ITP's proof commands correspond to standard inference rules present in ITP's. Then, they can be easily replaced by the proof commands present in a particular ITP.

As concerns proof automation, the drop command 


$\frac{\Gamma, f=\{(x, y),(x, z) \sqcup N\} \vdash y=z \quad \Gamma, f=\{v \sqcup N\} \vdash \operatorname{pair}(v)}{\Gamma \vdash \operatorname{pfun}(f)}$

FIGURE 4. Example of a rewrite step

plays a central role. In effect, it allows to call $\{\log \}$ with the minimal set of hypotheses as to prove the goal. This implies a reduction of the proof term and consequently of the computing time. As opposed to the usual fact that the more hypotheses are available during an interactive proof, the better, dropping hypotheses is decisive to the success of our approach. Indeed, when an ATP is called to perform a proof step, unnecessary hypotheses may make it walk through many actually useless proof paths (and, in the case of tools like $\{\log \}$, that might not terminate in some cases, they can take an infinite proof path). Hence, by dropping unnecessary hypotheses the prover has fewer proof paths to walk through, thus augmenting the chances to end the proof and to do it faster.

On the downside, the key role of drop sensibly changes the proof style as now the user must determine which hypotheses are superfluous to prove a subgoal instead of using them to prove it.

We also note that our approach tends to reduce the influence of a good lemma engineering. That is, users usually plan which lemmas go first and which follow, so as to use the former in the proofs of the latter. For example, the Coq proof of (T1) is the following:

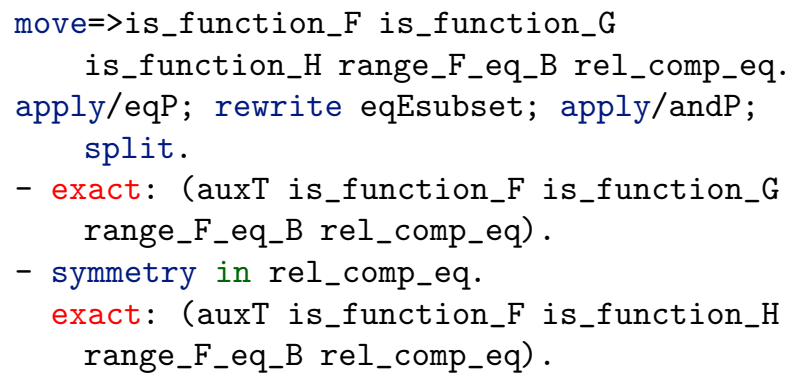

where auxT is a helper lemma whose importance was made evident after the first proof attempt (T1). Indeed, auxT states that $g \subseteq h$ holds if the hypotheses of (T1) are satisfied. Its proof is the following:

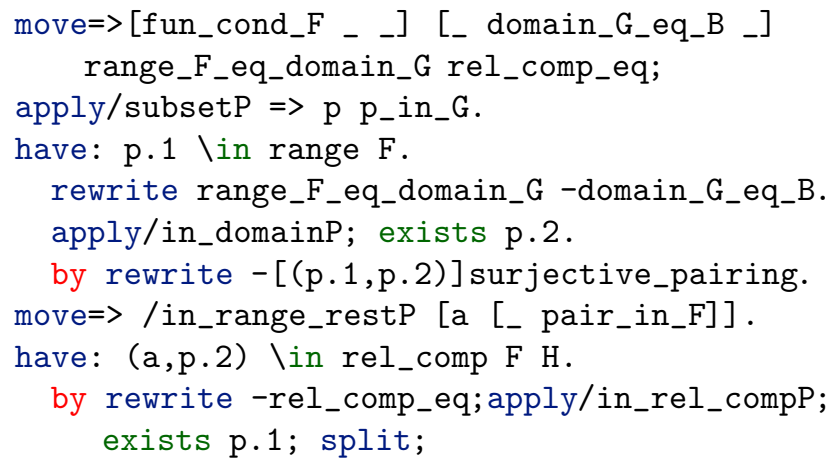

${ }^{5}$ The reader does not need to understand the proofs, just to have an idea of their length and complexity.

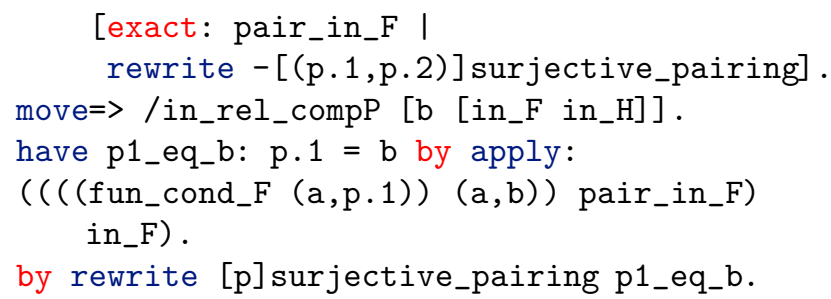

Now, compare Coq's proof of (T1) with $\{\log \}$-ITP's (cf. Section 3.1):

rewrite.

$$
\begin{aligned}
& \text { - } \operatorname{drop}([f \subseteq A \times B, \operatorname{dom}(f, A), \\
& \quad g \subseteq B \times C, \operatorname{dom}(g, B), h \subseteq B \times C]), \\
& \text { prove. } \\
& \text { - } \operatorname{drop}([f \subseteq A \times B, \operatorname{dom}(f, A), \\
& \quad g \subseteq B \times C, h \subseteq B \times C, \operatorname{dom}(h, B)]), \\
& \text { prove. }
\end{aligned}
$$

where no helper lemma is necessary and the complex proof of auxT is replaced by dropping hypotheses and then calling $\{\log \}$. On the other hand, as in Coq, the user still needs to guide the proof by splitting the initial goal into $g \subseteq h$ and $h \subseteq g$ and the symmetry used in the Coq proof (i.e., symmetry) is still present in the $\{\log \}$-ITP proof, when symmetric drop commands are executed. Hence, were $\{\log \}$ available in Coq the proof of (T1) would not need the helper lemma and it would still be compact and semi-automatic.

However, there is still room for further automation. CoqHammer [6] uses external ATPs to automate Coq proofs. CoqHammer helps in automating the proof of (T1):

move $=>$ is_function_F is_function_G is_function_H range_F_eq_B rel_comp_eq. apply/eqP; rewrite eqEsubset; apply/andP; split.

- hammer.

- hammer.

where hammer needs lemma auxT to prove both subgoals. However, hammer cannot prove auxT automatically. Then, were CoqHammer and $\{\log \}$ available, the Coq proof of (T1) could be almost automatic: first use drop and prove to prove auxT; and then use hammer to prove (T1) as above.

CoqHammer depends on a good lemma engineering. Conversely, $\{\log \}$ does not depend on such engineering but it can only prove results of FS\&RA. A combination between a general tool like CoqHammer with specialized provers such as $\{\log \}$, seems to be a promising strategy towards proof automation. 


\section{EMPIRICAL ASSESSMENT}

As we have said, our intention is to provide evidence that integrating $\{\log \}$ 's rewriting system into mainstream ITP's will yield a noticeable increment in proof automation concerning FS\&RA. This is $\{\log \}$ ITP's single purpose. To this end, we performed 210 proofs with $\{\log \}$-ITP and Coq in order to compare their complexity and length. In an attempt to avoid as much as possible a bias towards $\{\log \}$-ITP, 21 proofs correspond to problems listed in the REL and SET collections of the TPTP library ((T1) is an example) which satisfy that $\{\log \}$ either does not terminate or takes a very long time to do it when it is applied to prove them 6 . The remaining 189 proofs correspond to lemmas included in Coq's SSReflect finite set library, finset 39 . We chose finset for three reasons: a) it has been designed as to make it easy to prove those lemmas in Coq; b) a fragment of finset's set theory keeps a clear relationship with respect to $\{\log \}$ 's; and $c$ ) it would provide evidence that proof automation of real Coq results can be achieved with our proposal. Finally, the Coq proofs were performed by one of the authors (with experience in working with SSReflect), while $\{\log \}$-ITP's were done by another author.

As concerns the TPTP problems, they are encoded in Coq by extending SSReflect's finset. SSReflect is a proof language extending Coq with additional tactics oriented to support long mathematical proofs. finset defines a type for sets over a finite type. It includes definitions such as set membership, union, Cartesian product, etc. However, it does not include set relation algebra definitions such as the identity relation, converse (or inverse), composition, and (partial) function. Since these are necessary to reason about $\mathcal{L}_{\mathcal{B R}}$ formulas, we defined them in Coq. For example, our Coq set-based definition of function from $A$ to $B$ (i.e., $f \in A \rightarrow B$ ) is the following:

Definition is_function_from_to

(S1 S2:finType) (R: $\{\operatorname{set}(\mathrm{S} 1 * \mathrm{~S} 2)\})$

$(A:\{$ set $S 1\})(B:\{$ set $S 2\}):=$

[八 is_function $R$, domain $R=A$ \& (range R) \subset $B$ ] .

where is_function, domain and range are defined in a similar fashion, but where \subset is part of SSReflect's finite type interface (on which finset is based on). We also give a set-based definition of the relational composition of two binary relations:

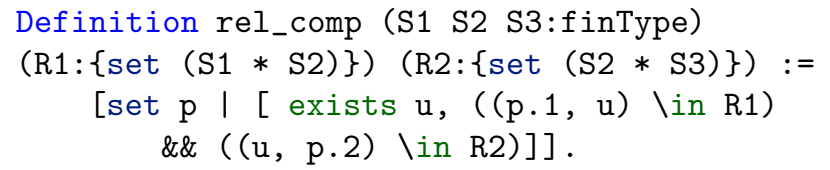

\footnotetext{
${ }^{6}\{\log \}$ automatically and quickly proves all the other problems of TPTP.SET and TPTP.REL expressible in its input language 10.

${ }^{7}$ The remaining 147 lemmas of finset are not expressible in the input language of $\{\log \}$ as they include operators such as
} generalized union or powerset.
These extensions to finset lead to the following encoding of theorem (T1):

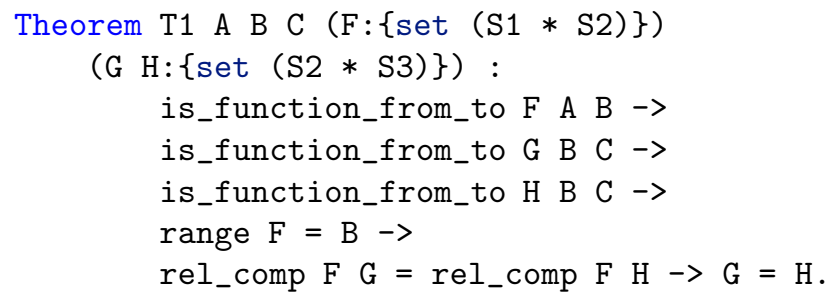

A similar encoding was used to state the 189 lemmas of finset, as $\{\log \}$-ITP theorems. For example, the following finset lemma:

Lemma setCU A B :

$\sim:(\mathrm{A}: \mid: \mathrm{B})=\sim: \mathrm{A}: \&: \sim: \mathrm{B}$

where ${ }^{\sim}$ : is complement $\left({ }^{-}\right),: \mid:$is $\cup$ and $: \&:$ is $\cap$, is encoded as the following $\{\log \}$-ITP theorem:

$$
\begin{aligned}
& \text { theorem } \\
& \qquad \begin{aligned}
& \text { setCU, } \\
& A \subseteq T \wedge B \subseteq T \wedge u n\left(A, B, M_{1}\right) \\
& \wedge u n\left(M_{1}, M_{2}, T\right) \wedge M_{1} \| M_{2} \wedge u n\left(A, M_{3}, T\right) \\
& \wedge A \| M_{3} \wedge \text { un }\left(B, M_{4}, T\right) \wedge B \| M_{4}, \\
& \text { inters } \left.\left(M_{3}, M_{4}, M_{2}\right)\right)
\end{aligned}
\end{aligned}
$$

where $T$ corresponds to the variable $T$ of type finType declared in the section containing Lemma setCU (i.e., all sets of this section are subsets of $\mathrm{T}$ ).

Table 1 summarizes the results of the evaluation. As we have said, $\{\log \}$ is unable to automatically prove any of the 21 TPTP theorems (thus, for the TPTP theorems, the Auto entry is set to zero). On the other hand, Coq needs 690 proof commands to prove them, while $\{\log \}$-ITP can do it with 219, of which only 146 are other than the prove command. This is a reduction of about $68 \%$ in the number of proof commands (and about $79 \%$ if prove is not counted). By 'proof command' we understand all the characters accommodated in the same line (which intend to represent basic mathematical proof steps). For example, for us, this is a single Coq proof command: by move $=>$ a; apply/setP $=>$ $\mathrm{x}$; rewrite inE; case: eqP $\Rightarrow \rightarrow$. In this sense, $\{\log \}$-ITP proof commands tend to be simpler than Coq's. Actually, the 690 proof commands used in Coq to prove the TPTP problems are composed of about 1030 applications of SSReflect tactics, which represent 35,014 characters while those used in $\{\log \}$-ITP just 3,662 characters. Then, apart from the gain in the number of proof commands, there is notable gain in their complexity $(\approx 90 \%)$. Finally, collectively all the prove commands consume $55 \mathrm{~s}$ of computing time which means that the automated part of each theorem is executed in $2.6 \mathrm{~s}$ in average. On the other hand, the completion of all the Coq proofs took around 10 manhour; while completing all the $\{l o g\}$-ITP took around 1 man-hour.

As concerns the problems taken from $\mathrm{f}$ inset, Table 1 indicates that $\{\log \}$-ITP automatically proves $97 \%$ of them (in $1 \mathrm{~s}$ in average). In this case the gain in the 


\begin{tabular}{|c|c|c|c|c|c|c|c|c|}
\hline \multicolumn{9}{|l|}{$\mathrm{PR}$} \\
\hline Collection & $\#$ & Auto & $\%$ & \multicolumn{3}{|c|}{ COMMANDS } & Computing Time & Avg Computing Time \\
\hline TPTP & 21 & 0 & 0 & 690 & 219 & 146 & $55 \mathrm{~s}$ & $2.6 \mathrm{~s}$ \\
\hline SSReflect finset & 189 & 183 & 97 & 223 & 195 & 6 & $182 \mathrm{~s}$ & $1 \mathrm{~s}$ \\
\hline SUMMARY & 210 & 183 & 87 & 913 & 435 & 158 & $237 \mathrm{~s}$ & $1.1 \mathrm{~s}$ \\
\hline
\end{tabular}

TABLE 1. Summary of the empirical evaluation

number of proof commands is minimal. However, it should be noted that in $\{\log \}$-ITP 183 problems are proved via the same command (prove), while the Coq proofs require, roughly, 214 different commands. In other words, a Coq user needs to reason on how to prove 183 theorems, while a $\{\log \}$-ITP user does not. This fact can be quantified if only the non-prove commands are considered, as they amount to only $2 \%$ of the Coq commands.

The Coq proofs present in these experiments are the result of some degree of lemma engineering. For instance, in the TPTP Coq proofs we first proved 21 helper lemmas and then the 21 theorems used as experiment. The helper lemmas correspond to properties that are used several times in the proofs of the 21 theorems. These can be simple properties, such as the characterization of the fact that an ordered pair belongs to the relational composition of two binary relations, or more complex ones, such as the helper lemma auxT described in Section 3.3 (which is applied twice in the proof of (T1) thanks to the use of symmetry). Without this lemma engineering, proofs would be longer and more complex. As usual, many of these helper lemmas make themselves evident after some of the main theorems are proved. In $\{\log \}$-ITP no lemma engineering was used (actually, there is no way to use or apply a previous lemma in the current proof). This is another indication of a gain in simplicity when $\{\log \}$-ITP is used.

This evaluation suggests that an integration of $\{\log \}$ into Coq would produce more fully automated FS\&RA proofs and would semi-automate many others.

The full data set of our experiments can be found online at:

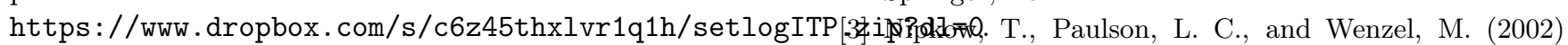
They were performed on a Dell Latitude E7470 (06DC) laptop with a 4 core Intel(R) $\operatorname{Core}^{\mathrm{TM}} \mathrm{i} 7-6600 \mathrm{U}$ CPU at $2.60 \mathrm{GHz}$ with $8 \mathrm{~Gb}$ of main memory, running Linux Ubuntu 18.04.2 LTS 64-bit with kernel 4.15.0-56generic. The following software versions were used: $\{\log \}$ 4.9.6-5d over SWI-Prolog (multi-threaded, 64 bits, version 7.6.4); Coq 8.9.0; CoqHammer 1.1 using Vampire 4.2.2, E prover 2.3, Z3 4.8.4.0 and CVC4 1.6.

\section{CONCLUSIONS}

We have proposed $\{l o g\}$ as a special purpose ATP for the theory of finite sets and finite set relation algebra, that can be integrated into ITPs to semi-automate proofs of this theory. We have also empirically evaluated the approach by implementing a prototype ITP where users can call $\{\log \}$ as a proof command. The prototype was assessed on 210 theorems and compared with Coq. The assessment shows good results in: $a$ ) the number of automated proofs; $b$ ) the computing times needed to complete them; and $c$ ) the reduction in the length and complexity of interactive proofs that call $\{\log \}$ to discharge subgoals.

Concerning future work, in the case of Coq we see two possible integration strategies. The most obvious one is to use $\{\log \}$ as an external ATP, along the lines of SMTCoq [8] or CoqHammer [6]. In this case proof reconstruction might be difficult or infeasible. A second integration strategy may consist in implementing $\{\log \}$ 's rewriting system as a Coq tactic. The first steps of this approach have already been done by Dubois and Weppe 40. Depending on the way this is done, as a side effect, this approach might yield a formal verification of $\{\log \}$. It remains as open issues, though, whether the size of the proof term produced by the new tactic will be more manageable than in the first strategy and whether or not the tactic will be fast enough as to be worth it.

\section{REFERENCES}

[1] Harrison, J., Urban, J., and Wiedijk, F. (2014) History of interactive theorem proving. In Siekmann, J. H. (ed.), Computational Logic, Handbook of the History of Logic, 9, pp. 135-214. Elsevier.

[2] Bertot, Y. and Castéran, P. (2004) Interactive Theorem Proving and Program Development - Coq'Art: The Calculus of Inductive Constructions Texts in Theoretical Computer Science. An EATCS Series. Springer, Berlin. Isabelle/HOL - A Proof Assistant for Higher-Order Logic, Lecture Notes in Computer Science, 2283. Springer, Berlin.

[4] Harrison, J. (1996) HOL light: A tutorial introduction. In Srivas, M. K. and Camilleri, A. J. (eds.), Formal Methods in Computer-Aided Design, First International Conference, FMCAD '96, Palo Alto, California, USA, November 6-8, 1996, Proceedings, Lecture Notes in Computer Science, 1166, pp. 265269. Springer, Berlin.

[5] Nieuwenhuis, R., Oliveras, A., and Tinelli, C. (2006) Solving SAT and SAT modulo theories: From an abstract Davis-Putnam-Logemann-Loveland procedure to DPLL(T). J. ACM, 53, 937-977.

[6] Czajka, L. and Kaliszyk, C. (2018) Hammer for coq: Automation for dependent type theory. J. Autom. 
Reasoning, 61, 423-453.

[7] Paulson, L. C. and Blanchette, J. C. (2010) Three years of experience with sledgehammer, a practical link between automatic and interactive theorem provers. In Sutcliffe, G., Schulz, S., and Ternovska, E. (eds.), The 8th International Workshop on the Implementation of Logics, IWIL 2010, Yogyakarta, Indonesia, October 9, 2011, EPiC Series in Computing, 2, pp. 1-11. EasyChair.

[8] Ekici, B., Mebsout, A., Tinelli, C., Keller, C., Katz, G., Reynolds, A., and Barrett, C. W. (2017) Smtcoq: A plug-in for integrating SMT solvers into Coq. In Majumdar, R. and Kuncak, V. (eds.), Computer Aided Verification - 29th International Conference, CAV 2017, Heidelberg, Germany, July 24-28, 2017, Proceedings, Part II, Lecture Notes in Computer Science, 10427, pp. 126-133. Springer, Berlin.

[9] Blanchette, J. C., Böhme, S., and Paulson, L. C. (2013) Extending Sledgehammer with SMT solvers. J. Autom. Reasoning, 51, 109-128.

[10] Cristiá, M. and Rossi, G. (2020) Solving quantifierfree first-order constraints over finite sets and binary relations. J. Autom. Reasoning, 64, 295-330.

[11] Jackson, D. (2006) Software Abstractions: Logic, Language, and Analysis. The MIT Press.

[12] Abrial, J.-R. (1996) The B-book: Assigning Programs to Meanings. Cambridge University Press, New York, NY, USA.

[13] Spivey, J. M. (1992) The $Z$ notation: a reference manual. Prentice Hall International (UK) Ltd., Hertfordshire, UK, UK.

[14] Sutcliffe, G. (2009) The TPTP Problem Library and Associated Infrastructure: The FOF and CNF Parts, v3.5.0. J. Autom. Reasoning, 43, 337-362.

[15] Cristiá, M. and Rossi, G. (2018) A set solver for finite set relation algebra. In Desharnais, J., Guttmann, W., and Joosten, S. (eds.), Relational and Algebraic Methods in Computer Science - 17th International Conference, RAMiCS 2018, Groningen, The Netherlands, October 29 - November 1, 2018, Proceedings, Lecture Notes in Computer Science, 11194, pp. 333-349. Springer, Berlin.

[16] Andréka, H., Givant, S. R., and Németi, I. (1997) Decision problems for equational theories of relation algebras. American Mathematical Society, Providence, Rhode Island, USA.

[17] Mentré, D., Marché, C., Filliâtre, J.-C., and Asuka, M. (2012) Discharging proof obligations from Atelier B using multiple automated provers. In Derrick, J., Fitzgerald, J. A., Gnesi, S., Khurshid, S., Leuschel, M., Reeves, S., and Riccobene, E. (eds.), $A B Z$, Lecture Notes in Computer Science, 7316, pp. 238251. Springer, Berlin.

[18] Bobot, F., Filliâtre, J.-C., Marché, C., and Paskevich, A. (2011) Why3: Shepherd your herd of provers. Boogie 2011: First International Workshop on Intermediate Verification Languages, Wrocław, Poland, August.

[19] Dénès, M., Hritcu, C., Lampropoulos, L., Paraskevopoulou, Z., and Pierce, B. C. (2014) Quickchick: Property-based testing for Coq. The Coq Workshop.
[20] Schulz, S. (2002) E - a brainiac theorem prover. AI Commun., 15, 111-126.

[21] Riazanov, A. and Voronkov, A. (2002) The design and implementation of VAMPIRE. AI Commun., 15, 91110.

[22] Blanchette, J. C., Böhme, S., Popescu, A., and Smallbone, N. (2016) Encoding monomorphic and polymorphic types. Logical Methods in Computer Science, 12, 1-52.

[23] Bury, G., Delahaye, D., Doligez, D., Halmagrand, P., and Hermant, O. (2015) Automated deduction in the $\mathrm{B}$ set theory using typed proof search and deduction modulo. In Fehnker, A., McIver, A., Sutcliffe, G., and Voronkov, A. (eds.), 20th International Conferences on Logic for Programming, Artificial Intelligence and Reasoning - Short Presentations, LPAR 2015, Suva, Fiji, November 24-28, 2015., EPiC Series in Computing, 35, pp. 42-58. EasyChair.

[24] Bury, G., Cruanes, S., Delahaye, D., and Euvrard, P. (2018) An automation-friendly set theory for the B method. In Butler, M. J., Raschke, A., Hoang, T. S., and Reichl, K. (eds.), Abstract State Machines, Alloy, B, TLA, VDM, and Z - 6th International Conference, ABZ 2018, Southampton, UK, June 5-8, 2018, Proceedings, Lecture Notes in Computer Science, 10817, pp. 409-414. Springer, Berlin.

[25] Conchon, S. and Contejean, E. Alt-Ergo. last access: November 2011.

[26] Bury, G. and Delahaye, D. ArchSat. last access: October 2019.

[27] Cruanes, S. Zipperposition. last access: October 2019.

[28] Cristiá, M. and Rossi, G. (2016) A decision procedure for sets, binary relations and partial functions. In Chaudhuri, S. and Farzan, A. (eds.), Computer Aided Verification - 28th International Conference, CAV 2016, Toronto, ON, Canada, July 17-23, 2016, Proceedings, Part I, Lecture Notes in Computer Science, 9779, pp. 179-198. Springer, Berlin.

[29] Rossi, G. (2008). $\{\log \}$. http://people.dmi.unipr.it/gianfranco.rossi/setlog.Home.html

[30] Cristiá, M., Rossi, G., and Frydman, C. (2017) Using a set constraint solver for program verification. Proceedings 4th Workshop on Horn Clauses for Verification and Synthesis, HCVS at CADE 2017, Gothenburg, Sweden, 7th August 2017.

[31] Cristiá, M., Rossi, G., and Frydman, C. S. (2013) $\{\log \}$ as a test case generator for the Test Template Framework. In Hierons, R. M., Merayo, M. G., and Bravetti, M. (eds.), SEFM, Lecture Notes in Computer Science, 8137, pp. 229-243. Springer, Berlin.

[32] Cristiá, M. and Rossi, G. (2014) Rapid prototyping and animation of $\mathrm{Z}$ specifications using $\{\log \}$. 1 st International Workshop about Sets and Tools (SETS 2014), pp. 4-18. Informal proceedings: http://sets2014.cnam.fr/papers/sets2014.pdf

[33] Schmidt, G., Hattensperger, C., and Winter, M. (1997) Heterogeneous Relation Algebra. In Brink, C., Kahl, W., and Schmidt, G. (eds.), Relational Methods in Computer Science. Springer Vienna, Vienna.

[34] Cristiá, M. and Rossi, G. (2020) Automated proof of Bell-LaPadula security properties. J. Autom. Reasoning, $\mathbf{n / a . ~}$ 
[35] Cristiá, M. and Rossi, G. (2020) An automatically verified prototype of the Tokeneer ID station specification. CoRR, abs/2009.00999.

[36] Dovier, A., Piazza, C., Pontelli, E., and Rossi, G. (2000) Sets and constraint logic programming. ACM Trans. Program. Lang. Syst., 22, 861-931.

[37] Cristiá, M. and Rossi, G. (2019). Rewrite rules for a solver for sets, binary relations and partial functions.

[38] Cristiá, M. (2019). $\quad\{\log \}$-ITP source code and experimental data.

[39] Gonthier, G. and Mahboubi, A. (2010) An introduction to small scale reflection in Coq. J. Formalized Reasoning, 3, 95-152.

[40] Dubois, C. and Weppe, S. (2018) Towards Coq formalisation of $\{\log \}$ set constraints resolution. In Cristiá, M., Delahaye, D., and Dubois, C. (eds.), Proceedings of the 3rd International Workshop on Sets and Tools co-located with the 6th International ABZ Conference, SETS@ABZ 2018, Southamptom, UK, June 5, 2018., CEUR Workshop Proceedings, 2199, pp. 32-37. CEUR-WS.org.

\section{APPENDIX A. SYNTAX AND SEMANTICS OF $\mathcal{L}_{\mathcal{B R}}$}

In this appendix we provide a formal, detailed introduction of the syntax and semantics of $\mathcal{L}_{\mathcal{B R}}$.

The input constraint language accepted by $\{\log \}$, $\mathcal{L}_{\mathcal{B R}}$, is a first-order predicate language with terms of two sorts: terms designating sets (including binary relations), and terms designating ur-elements. Terms of either sort are allowed to enter in the formation of set terms (in this sense, the designated sets are hybrid), no nesting restrictions being enforced (in particular, membership chains of any finite length can be modeled). In a term which is not a variable and designates an ur-element, the main functor (be it a constant or a function symbol) will act as a free ('uninterpreted') Herbrand constructor; a special set constructor, and a handful of reserved predicate symbols endowed with a pre-designated set-theoretic meaning, are also available. Formulas are built in the usual way by using conjunction, disjunction and negation of atomic predicates. A number of complex operators (in the form of predicates) are defined as $\mathcal{L}_{\mathcal{B} \mathcal{R}}$ formulas, thus making it simpler for the user to write complex formulas.

\section{Appendix A.1. Syntax}

The syntax of $\mathcal{L}_{\mathcal{B R}}$ is defined primarily by giving the signature upon which terms and formulas are built.

Definition A.1. The signature $\Sigma_{\mathcal{B R}}$ of $\mathcal{L}_{\mathcal{B R}}$ is a tuple $\langle\mathcal{F}, \Pi$, Set, $\mathrm{O}, \mathcal{V}\rangle$ where:

- $\mathcal{F}$ is the set of function symbols partitioned as $\mathcal{F} \hat{=}$ $\mathcal{F}_{S} \cup \mathcal{F}_{\mathcal{X}}$, where $\mathcal{F}_{S} \hat{=}\{\emptyset,\{\cdot \sqcup \cdot\}, \cdot \times \cdot\}$ and $\mathcal{F}_{\mathcal{X}}$ is a set of uninterpreted constant and function symbols, including at least the binary function symbol $(\cdot, \cdot)$.
- $\Pi$ is the set of predicate symbols partitioned as $\Pi \widehat{=} \Pi_{S} \cup \Pi_{T} \cup \Pi_{R}$, where $\Pi_{S} \widehat{=}\{=, \neq, \in, \notin$, un, $\|\}$, $\Pi_{T} \widehat{=}\{$ set, nset, rel, nrel, pair, npair $\}$ and $\Pi_{R} \widehat{=}$ $\{i d$, comp, inv $\}$.

- $\{$ Set, $\mathrm{O}\}$ is the set of sorts.

- $\mathcal{V}$ is a denumerable set of variables partitioned as $\mathcal{V} \hat{=} \mathcal{V}_{S} \cup \mathcal{V}_{O}$, where $\mathcal{V}_{S}$ and $\mathcal{V}_{O}$ contain variables of sort Set and $\mathrm{O}$, respectively.

To complete the definition of $\mathcal{L}_{\mathcal{B R}}$, in addition to the signature it is necessary to specify the sorts of function and predicate symbols: if $f \in \mathcal{F}$ (resp., $\pi \in \Pi$ ) is of arity $n$, then its sort is an $n+1$-tuple $\left\langle s_{1}, \ldots, s_{n+1}\right\rangle$ (resp., an $n$-tuple $\left\langle s_{1}, \ldots, s_{n}\right\rangle$ ) of non-empty subsets of the set of sorts $\{$ Set, $\mathrm{O}\}$. This notion is denoted by $f:\left\langle s_{1}, \ldots, s_{n+1}\right\rangle$ (resp., by $\pi:\left\langle s_{1}, \ldots, s_{n}\right\rangle$ ).

Definition A.2. The sorts of the function symbols in $\mathcal{F}$ are as follows:

- $\emptyset:\langle\{$ Set $\}\rangle$;

- $\{\cdot \sqcup \cdot\}:\langle\{$ Set, O $\},\{$ Set $\},\{$ Set $\}\rangle$;

$\bullet \times \cdot:\langle\{$ Set $\},\{$ Set $\},\{$ Set $\}\rangle$;

- $(\cdot, \cdot):\langle\{$ Set, O $\},\{$ Set, O $\},\{O\}\rangle$;

- $f:\langle\{\mathrm{O}\}, \ldots,\{\mathrm{O}\}\rangle \in(\{\mathrm{O}\})^{n+1}$ if $f \in \mathcal{F}_{\mathcal{X}}$ is of arity $n$.

The sorts of the predicate symbols in $\Pi$ are as follows (symbols $=, \neq, \in, \notin$ and $\|$ are infix; all other symbols in $\Pi$ are prefix):

- pair, npair, set, nset : $\langle\{$ Set, $\mathrm{O}\}\rangle$;

- $=, \neq:\langle\{$ Set, O) $\},\{$ Set, O $\}\rangle$;

- $\in, \notin:\langle\{$ Set, O\}, $\{$ Set $\}\rangle$;

- un, comp : $\langle\{$ Set $\},\{$ Set $\},\{$ Set $\}\rangle$;

- $\|, i d$, inv : $\langle\{$ Set $\},\{$ Set $\}\rangle$;

- $r e l$, nrel : $\langle\{$ Set $\}\rangle$.

We can now define the set of admissible (i.e., wellsorted) $\mathcal{L}_{\mathcal{B R}}$ terms.

Definition A.3. All $\mathcal{B R}$-terms and their sorts are build inductively as follows:

- each variable $v \in V$ is a $\mathcal{B R}$-term of sort $\langle\{$ Set $\}\rangle$ if $v \in \mathcal{V}_{S}$ or sort $\langle\{O\}\rangle$ if $v \in \mathcal{V}_{O}$.

- if $f \in \mathcal{F}$ is a function symbol of sort $\left\langle s_{1}, \ldots, s_{n+1}\right\rangle$, and for each $i=1, \ldots, n, t_{i}$ is a $\mathcal{B R}$-term of sort $\left\langle s_{i}^{\prime}\right\rangle$ with $s_{i}^{\prime} \subseteq s_{i}$, then $f\left(t_{1}, \ldots, t_{n}\right)$ is a $\mathcal{B R}$-term of sort $\left\langle s_{n+1}\right\rangle$.

Note that the sort of any $\mathcal{B R}$-term $t$ is always of the form $\langle\{$ Set $\}\rangle$ or $\langle\{O\}\rangle$. In the former case we simply say that $t$ is of sort Set, or a set term, and in the latter case that $t$ is of sort $\mathrm{O}$. In particular, $\mathcal{B R}$-terms of the form $\{\cdot \sqcup \cdot\}$ are called extensional set terms. The first parameter of an extensional set term is called element part and the second is called set part. Observe that one can write terms representing sets which are nested at any level.

The following notation is introduced to make reading of set terms simpler: $\left\{t_{1}, t_{2}, \ldots, t_{n} \sqcup t\right\}$ as a shorthand 
for $\left\{t_{1} \sqcup\left\{t_{2} \sqcup \cdots\left\{t_{n} \sqcup t\right\} \cdots\right\}\right\}$ and the notation $\left\{t_{1}, t_{2}, \ldots, t_{n}\right\}$ as a shorthand for $\left\{t_{1}, t_{2}, \ldots, t_{n} \sqcup \emptyset\right\}$.

ExAmple 3 . The following are set terms:

$-\emptyset$

- $\{a,(b, c)\}$, i.e., $\{a \sqcup\{(b, c) \sqcup \emptyset\}\}$, where $a, b$ and $c$ are constants of sort $\mathrm{O}$

- $\{x \sqcup A \times\{y \sqcup B\}\}$, where $x, y$ are variables of sort Set or $\mathrm{O}$, and $A, B$ are variables of sort Set.

- $\{x \sqcup A\}$, where $x$ is a variable of sort Set or $\mathrm{O}$, and $A$ is a variable of sort Set.

On the opposite, $\{x \sqcup(a, b)\}$ is not a set term because $(a, b)$ is not of sort Set.

Finally, from $\mathcal{L}_{\mathcal{B R}}$ terms, we define $\mathcal{L}_{\mathcal{B R}}$ formulas.

Definition A.4. All $\mathcal{B R}$-formulas are build inductively as follows:

- if $\pi \in \Pi$ is a predicate symbol of sort $\left\langle s_{1}, \ldots, s_{n}\right\rangle$, and for each $i=1, \ldots, n, t_{i}$ is a $\mathcal{B R}$-term of sort $\left\langle s_{i}^{\prime}\right\rangle$ with $s_{i}^{\prime} \subseteq s_{i}$, then $\pi\left(t_{1}, \ldots, t_{n}\right)$ is a $\mathcal{B R}$ constraint, a particular case of $\mathcal{B R}$-formula.

- if $\alpha$ and $\beta$ are $\mathcal{B R}$-formulas, then so are $\alpha \wedge \beta$ and $\alpha \vee \beta$.

EXAMPLE 4 . The following are $\mathcal{B R}$-formulas:

$$
\begin{aligned}
& a \in A \wedge a \notin B \wedge u n(A, B, C) \wedge C=\{x\} \\
& u n(A, B, C) \wedge A \| C \wedge \operatorname{inv}(R, A) \wedge R \neq \emptyset
\end{aligned}
$$

where $a$ is a constant of sort $\mathrm{O}, x$ is a variable of sort Set or $\mathrm{O}$, and $A, B, C$ and $R$ are variables of sort Set. On the contrary, un $(A, B,(x, y))$ is not a $\mathcal{B R}$-formula because $u n(A, B,(x, y))$ is not a $\mathcal{B R}$-constraint $((x, y)$ is not of sort Set as required by the sort of un).

\section{Appendix A.2. Semantics}

Semantics of $\mathcal{B R}$-formulas is given by defining a suitable interpretation structure for $\mathcal{L}_{\mathcal{B R}}$.

Sorts and symbols in $\Sigma_{\mathcal{B R}}$ are interpreted according to the interpretation structure $\mathcal{R} \widehat{=}\left\langle D,(\cdot)^{\mathcal{R}}\right\rangle$, where $D$ and $(\cdot)^{\mathcal{R}}$ are defined as follows.

Definition A.5 (Interpretation domain). The interpretation domain $D$, of the interpretation structure $\mathcal{R}$, is partitioned as $D \hat{=} D_{\text {Set }} \cup D_{\mathrm{O}}$ where:

- $D_{\text {Set }}$ is the collection of all hereditarily finite hybrid sets built from elements in $D$; and

- $D_{\mathrm{O}}$ is a collection of other objects, including ordered pairs of elements in $D$.

Hereditarily finite sets are those sets that admit (hereditarily finite) sets as their elements. Note that, finite binary relations and functions, as defined in Note 2 belong to $D_{\text {Set }}$.

Definition A.6 (Interpretation function). The interpretation function $(\cdot)^{\mathcal{R}}$, of the interpretation structure $\mathcal{R}$, is defined as follows.
- Each sort $\mathrm{S} \in\{\mathrm{Set}, \mathrm{O}\}$ is mapped to the domain $D_{\mathrm{S}}$.

- For each sort $\mathrm{S} \in\{\mathrm{Set}, \mathrm{O}\}$, each variable $x$ of sort $\mathrm{S}$ is mapped to an element $x^{\mathcal{R}}$ in $D_{\mathrm{S}}$.

The constant and function symbols in $\mathcal{F}_{S}$ are interpreted as follows:

- $\emptyset$ as the empty set;

- $\{x \sqcup A\}$ as the set $\left\{x^{\mathcal{R}}\right\} \cup A^{\mathcal{R}}$; and

- $A \times B$ as the set $A^{\mathcal{R}} \times B^{\mathcal{R}}$.

The predicate symbols in $\Pi$ are interpreted as follows:

- $x=y$ as $x^{\mathcal{R}}=y^{\mathcal{R}}$;

- $x \in A$ as $x^{\mathcal{R}} \in A^{\mathcal{R}}$;

- un $(A, B, C)$ as $C^{\mathcal{R}}=A^{\mathcal{R}} \cup B^{\mathcal{R}}$;

- $A \| B$ as $A^{\mathcal{R}} \cap B^{\mathcal{R}}=\emptyset$;

- $\operatorname{set}(x)$ as $x^{\mathcal{R}} \in D_{\text {Set }}$;

- $\operatorname{pair}(x)$ as $x^{\mathcal{R}} \in\{(a, b): a, b \in D\}$;

- $\operatorname{rel}(R)$ as $R^{\mathcal{R}} \subset\{(a, b): a, b \in D\}$;

- $i d(A, R)$ as $R^{\mathcal{R}}=\mathrm{id} A^{\mathcal{R}}$;

- $\operatorname{inv}(R, S)$ as $S^{\mathcal{R}}=\left(R^{\mathcal{R}}\right)^{\smile}$;

- $\operatorname{comp}(R, S, T)$ as $T^{\mathcal{R}}=R^{\mathcal{R}} \circ S^{\mathcal{R}} ;$ and

- any symbol $\pi^{\prime}$ in $\{\neq, \notin$, nrel, nset, npair $\} \quad$ is interpreted as $\neg \pi$ for the corresponding symbol $\pi$ in $\{=, \in$, rel, set,pair $\}$, where $\neg$ is logical negation.

The interpretation structure $\mathcal{R}$ is used to evaluate each $\mathcal{R} \mathcal{I} \mathcal{S}$-formula $\Phi$ into a truth value $\Phi^{\mathcal{R}}=$ $\{$ true, false $\}$ in the following way: $\mathcal{R} \mathcal{I} \mathcal{S}$-constraints are evaluated by $(\cdot)^{\mathcal{R}}$ according to the meaning of the corresponding predicates in set theory as defined above; $\mathcal{R I S}$-formulas are evaluated by $(\cdot)^{\mathcal{R}}$ according to the rules of propositional logic.

In particular, observe that equality between two set terms is interpreted as the equality in $D_{\text {Set }}$; that is, as set equality between hereditarily finite hybrid sets. Such equality is regulated by the standard extensionality axiom, which has been proved to be equivalent, for hereditarily finite sets, to the following equational axioms [36]:

$$
\begin{aligned}
& \{x, x \sqcup A\}=\{x \sqcup A\} \\
& \{x, y \sqcup A\}=\{y, x \sqcup A\} .
\end{aligned}
$$

Note $3 . \mathcal{L}_{\mathcal{B R}}$ can be extended to support other set and relational operators definable by means of suitable $\mathcal{L}_{\mathcal{B R}}$ formulas. Dovier et al. 36 proved that symbols in $\Pi_{S}$ are sufficient to define constraints implementing the set operators $\cap, \subseteq$ and $\backslash$. Cristiá and Rossi extend that result in 10] showing that symbols in $\Pi_{S} \cup \Pi_{R}$ are sufficient to define constraints implementing all the operators defined in Note 2

As any of these constraints can be replaced by its definition, we can completely ignore the presence of them in $\mathcal{L}_{\mathcal{B R}}$ formulas.

Note 4 (Negation). The negated versions of both set and relational constraints can be introduced as $\mathcal{L}_{\mathcal{B R}}$ 
formulas [36, 10]. For example, $\neg R=S^{\smile}$ is introduced as the following $\mathcal{B R}$-formula:

$$
\begin{aligned}
& ((x, y) \in R \wedge(y, x) \notin S) \vee((x, y) \notin R \wedge(y, x) \in S) \\
& \vee \operatorname{nrel}(R) \vee \operatorname{nrel}(S)
\end{aligned}
$$

( $x$ and $y$ are implicitly existentially quantified). Thanks to the availability of negative constraints, (general) logical negation is not strictly necessary in $\mathcal{L}_{\mathcal{B R}}$.

Data Availability Statement The data underlying this article are available in Dropbox at https://www .dropbox.com/s/c6z45thxlvr1q1h/setlogITP. $z i p ? d l=0$, and can be accessed with the URL just given. 